OPEN ACCESS

Edited by:

Toshio Hattori,

Kibi International University, Japan

Reviewed by:

Carmen Judith Serrano,

Mexican Social Security

Institute, Mexico

Luiz Gustavo Gardinassi,

Universidade Federal de Goiás (IPTSP

- UFG), Brazil

*Correspondence:

Gokul Raj Kathamuthu gokul.r@nirt.res.in

Specialty section:

This article was submitted to

Microbial Immunology,

a section of the journal

Frontiers in Immunology

Received: 05 December 2019

Accepted: 24 February 2020

Published: 11 March 2020

Citation:

Kathamuthu GR, Kumar NP

Moideen K, Nair D, Banurekha W,

Sridhar R, Baskaran D and Babu S

(2020) Matrix Metalloproteinases and

Tissue Inhibitors of Metalloproteinases Are Potential Biomarkers of Pulmonary

and Extra-Pulmonary Tuberculosis.

Front. Immunol. 11:419.

doi: 10.3389/fimmu.2020.00419

\section{Matrix Metalloproteinases and Tissue Inhibitors of Metalloproteinases Are Potential Biomarkers of Pulmonary and Extra-Pulmonary Tuberculosis}

\author{
Gokul Raj Kathamuthu ${ }^{1,2 *}$, Nathella Pavan Kumar ${ }^{1}$, Kadar Moideen ${ }^{1}$, Dina Nair ${ }^{2}$, \\ Vaithilingam V. Banurekha ${ }^{2}$, Rathinam Sridhar ${ }^{3}$, Dhanaraj Baskaran ${ }^{2}$ and Subash Babu ${ }^{1,4}$ \\ ${ }^{1}$ National Institute for Research in Tuberculosis, National Institute of Health, International Center for Excellence in Research, \\ Chennai, India, ${ }^{2}$ National Institute for Research in Tuberculosis (NIRT), Chennai, India, ${ }^{3}$ Government Stanley Medical \\ Hospital, Chennai, India, ${ }^{4}$ Laboratory of Parasitic Diseases, National Institute of Allergy and Infectious Diseases, National \\ Institutes of Health, Bethesda, MD, United States
}

Matrix metalloproteinases (MMPs) and tissue inhibitors of metalloproteinase (TIMPs) are potential regulators of tuberculosis (TB) pathology. Whether they are candidates for non-sputum-based biomarkers for pulmonary TB (PTB) and extra-pulmonary TB (EPTB) is not fully understood. Hence, to examine the association of MMPs and TIMPs with PTB and EPTB, we have measured the circulating levels of MMPs (MMP-1, 2, 3, 7, 8, 9, 12, and 13) and TIMPs (TIMP-1, 2, 3, and 4) in PTB, EPTB and compared them with latent tuberculosis (LTB) or healthy control $(\mathrm{HC})$ individuals. We have also assessed their circulating levels before and after the completion of anti-tuberculosis treatment (ATT). Our data describes that systemic levels of MMP-1, 8, 9, 12 were significantly increased in PTB compared to EPTB, LTB, and HC individuals. In contrast, MMP-7 was significantly reduced in PTB compared to EPTB individuals. Likewise, the systemic levels of MMP-1, 7, 13 were significantly increased in EPTB in comparison to LTB and HC individuals. In contrast, MMP-8 was significantly reduced in EPTB individuals compared to LTB and $\mathrm{HC}$ individuals. In addition, the systemic levels of TIMP-1, 2, 3 were significantly diminished and TIMP-4 levels were significantly enhanced in PTB compared to EPTB, LTB, and HC individuals. The circulating levels of TIMP-2 was significantly reduced and TIMP-3 was significantly elevated in EPTB individuals in comparison with LTB and HCs. Some of the MMPs $(7,8,9,12,13$ in PTB and 1, 7, 8, 9 in EPTB) and TIMPs (1, 2, 3,4 in PTB and 4 in EPTB) were significantly modulated upon treatment completion. ROC analysis showed that MMP-1, 9 and TIMP-2, 4 could clearly discriminate PTB from EPTB, LTB and HCs and MMP-13 and TIMP-2 could clearly discriminate EPTB from LTB and HCs. Additionally, multivariate analysis also indicated that these alterations were independent of age and sex in PTB and EPTB individuals. Therefore, our data demonstrates that MMPs and TIMPs are potential candidates for non-sputum-based biomarkers for differentiating PTB and EPTB from LTB and HC individuals.

\footnotetext{
Keywords: tuberculous lymphadenitis, MMPs, TIMPs, biomarkers, pulmonary tuberculosis
} 


\section{INTRODUCTION}

Mycobacterium tuberculosis (Mtb) kills nearly 1.5 million people globally and still poses a major threat with $90 \%$ of the disease occurring in developing countries $(1,2)$. Depending upon the Mtb exposure, infected individuals progress to a wide array of disease manifestations from symptomless latent TB (LTB) to active pulmonary TB (PTB) or extrapulmonary TB (EPTB) (3). Both PTB and EPTB suffer from diagnostic difficulties with low sensitivity of current diagnostic tests $(4,5)$. Most of the TB diagnostics depends upon the detection of pathogenic bacteria in sputum by culture, microscopy, or polymerase chain reaction (PCR) based assays like GeneXpert. However, difficulties do arise due to insufficient sputum collection, presence of few bacilli (paucibacillary) or extrapulmonary form of TB infection (6). Although the mortality rate of TB disease has reduced by $42 \%$ between the year 2000 and 2018, 3 million individuals are still undiagnosed or missed according to the World Health Organization (WHO) (7).

A systematic review in the year 2017 has shown that out of 399 biomarkers studied, only one urine-based biomarker (LAM, lipoarabinomannan) was considered as valid by WHO. However, LAM has minimal sensitivity [45\%] and moderate specificity [56\%] (8-10). Hence, it is essential to discover a rapid peripheral biomarker with a non-sputum test for diagnosis of pulmonary or extra-pulmonary TB and to distinguish between EPTB, PTB and LTB infected individuals $(11,12)$. Perhaps highpriority biomarkers with greater sensitivity $\geq 95 \%$ and specificity $>75 \%$ to rule out or differentiate the disease status should be given as the second highest priority. Therefore, identification of new biomarkers should provide detailed information on disease pathogenesis with adequate predictive value for clinical use.

Matrix metalloproteinases (MMPs) are enzymes responsible for tissue destruction, disease spread and mortality (13). MMPs belongs to the class of membrane bound zinc-binding endopeptidases and are highly proficient in degrading the extracellular matrix and basement membrane $(14,15)$. Diverse forms of MMP have been characterized in vertebrates [twenty eight, 28 forms] and humans [twenty four, 24 forms], which carry out several essential functions. Tissue inhibitors of metalloproteinases (TIMPs) comprise a family of 4 homologous secreted (TIMP-1, 2, 3, 4) proteins (16). TIMPs are very important factors for TB disease, involved in tissue remodeling and repair upon destruction created by MMPs $(17,18)$. Previous studies have identified MMPs as markers of disease severity, bacterial burdens and as a biomarker for disease in PTB and EPTB (17, 19-21). Relatively, few studies have focused on examining the circulating levels of MMPs and TIMPs as immune biomarkers in both PTB and EPTB.

We show that the systemic levels of MMPs and TIMPs were different between PTB and EPTB disease compared to the other study (LTB and HC) groups. In addition, we have also observed significant discrimination among various MMPs (1, 9 for PTB and 13 for EPTB) and TIMPs (2, 4 for PTB, and 2 for EPTB) between the study groups upon ROC analysis. Therefore, we suggest that combinations of MMPs and TIMPs could be potential candidates for non-sputum-based biomarkers in discriminating PTB from EPTB and PTB and EPTB from LTB and $\mathrm{HC}$ individuals.

\section{RESULTS}

\section{Altered Circulating Levels of MMPs in PTB and EPTB Individuals}

We measured the circulating levels of MMPs (MMP-1, 2, 3, 7, 8, 9, 12, and 13) in PTB, EPTB, LTB, and HC individuals (Figure 1). The systemic levels of MMP-1 (geometric mean (GM) of PTB is $1522 \mathrm{pg} / \mathrm{ml}$ vs. GM of EPTB is $202.2 \mathrm{pg} / \mathrm{ml}$ vs. GM of LTB is $64.03 \mathrm{pg} / \mathrm{ml}$ and $61.36 \mathrm{pg} / \mathrm{ml}$ in $\mathrm{HC}$ ), MMP-8 (GM of PTB is $4,722 \mathrm{pg} / \mathrm{ml}$ vs. GM of EPTB is $495.5 \mathrm{pg} / \mathrm{ml}$ vs. GM of LTB is $1,283 \mathrm{pg} / \mathrm{ml}$ and $1,342 \mathrm{pg} / \mathrm{ml}$ in HC), MMP-9 (GM of PTB is $9,270 \mathrm{pg} / \mathrm{ml}$ vs. GM of EPTB is $558.3 \mathrm{pg} / \mathrm{ml}$ vs. GM of LTB is $1,088 \mathrm{pg} / \mathrm{ml}$ and $1,171 \mathrm{pg} / \mathrm{ml}$ in HC) and MMP-12 (GM of PTB is $266.1 \mathrm{pg} / \mathrm{ml}$ vs. GM of EPTB is $204.7 \mathrm{pg} / \mathrm{ml}$ vs. GM of LTB is $206.6 \mathrm{pg} / \mathrm{ml}$ and $198.4 \mathrm{pg} / \mathrm{ml}$ in HC) were significantly higher in PTB individuals compared to EPTB, LTB, and HC individuals. In contrast, the circulating levels of MMP-7 (GM of PTB is 754.3 $\mathrm{pg} / \mathrm{ml}$ vs. GM of EPTB is $987 \mathrm{pg} / \mathrm{ml}$ vs. GM of LTB is $348.6 \mathrm{pg} / \mathrm{ml}$ and GM of $\mathrm{HC}$ is $586.9 \mathrm{pg} / \mathrm{ml}$ ) was significantly lower in PTB individuals compared to EPTB individuals.

As shown in Figure 1, the circulating levels of MMP-1 (GM of EPTB is $202.2 \mathrm{pg} / \mathrm{ml}$ vs. $64.03 \mathrm{pg} / \mathrm{ml}$ in LTB and $61.36 \mathrm{pg} / \mathrm{ml}$ in $\mathrm{HC}$ ), MMP-7 (GM of EPTB is $987 \mathrm{pg} / \mathrm{ml}$ vs. $348.6 \mathrm{pg} / \mathrm{ml}$ in LTB and $586.9 \mathrm{pg} / \mathrm{ml}$ in HC) and MMP-13 (GM of EPTB is $182.3 \mathrm{pg} / \mathrm{ml}$ vs. $97.97 \mathrm{pg} / \mathrm{ml}$ in LTB and $94.21 \mathrm{pg} / \mathrm{ml}$ in HC) were significantly higher in EPTB individuals when compared to LTB and $\mathrm{HC}$ individuals. In contrast, the circulating levels of MMP-8 (GM of EPTB is $495.5 \mathrm{pg} / \mathrm{ml}$ vs. $1,283 \mathrm{pg} / \mathrm{ml}$ in LTB and 1,342 $\mathrm{pg} / \mathrm{ml}$ in $\mathrm{HC}$ ) was significantly lower in EPTB compared to LTB and HC individuals. Thus, both PTB and EPTB are associated with altered plasma levels of MMPs.

\section{Altered Circulating Levels of TIMPs in PTB and EPTB Individuals}

We measured the circulating levels of TIMPs (TIMP-1, 2, 3, 4) in PTB, EPTB, LTB, and HC individuals. As shown in Figure 2, TIMP-1 (GM of PTB is $14,720 \mathrm{pg} / \mathrm{ml}$ vs. $19,959 \mathrm{pg} / \mathrm{ml}$ in ЕРТВ vs. $22,462 \mathrm{pg} / \mathrm{ml}$ in LTB and $20,596 \mathrm{pg} / \mathrm{ml}$ in HC) and TIMP2 (GM of PTB is $1,173 \mathrm{pg} / \mathrm{ml}$, GM of EPTB is $31,037 \mathrm{pg} / \mathrm{ml}$ vs. $19,249 \mathrm{pg} / \mathrm{ml}$ in LTB and 19,129 pg/ml in HC) and TIMP-3 (GM of PTB is $156.6 \mathrm{pg} / \mathrm{ml} \mathrm{GM}$ of EPTB is $91.71 \mathrm{pg} / \mathrm{ml}$ vs. $350.4 \mathrm{pg} / \mathrm{ml}$ in LTB and $312.2 \mathrm{pg} / \mathrm{ml}$ in $\mathrm{HC}$ ) and levels were significantly lower in PTB individuals compared to EPTB, LTB and HC individuals. In contrast, TIMP-4 levels were significantly higher in PTB (GM of PTB is $312.4 \mathrm{pg} / \mathrm{ml} \mathrm{GM}$ of EPTB is $34.19 \mathrm{pg} / \mathrm{ml}$ vs. $45.06 \mathrm{pg} / \mathrm{ml}$ in LTB and $31.69 \mathrm{pg} / \mathrm{ml}$ in HC) compared to EPTB, LTB, and HC individuals.

The circulating levels of TIMP-2 (GM of EPTB is 31,037 $\mathrm{pg} / \mathrm{ml}$ vs. $19,249 \mathrm{pg} / \mathrm{ml}$ in LTB and $19,129 \mathrm{pg} / \mathrm{ml}$ in HC) was significantly higher in EPTB individuals in comparison with LTB and $\mathrm{HC}$ individuals. In contrast, the systemic levels of TIMP-3 (GM of EPTB is $91.71 \mathrm{pg} / \mathrm{ml}$ vs. $350.4 \mathrm{pg} / \mathrm{ml}$ in LTB and 312.2 $\mathrm{pg} / \mathrm{ml}$ in $\mathrm{HC}$ ) was significantly lower in EPTB individuals in 


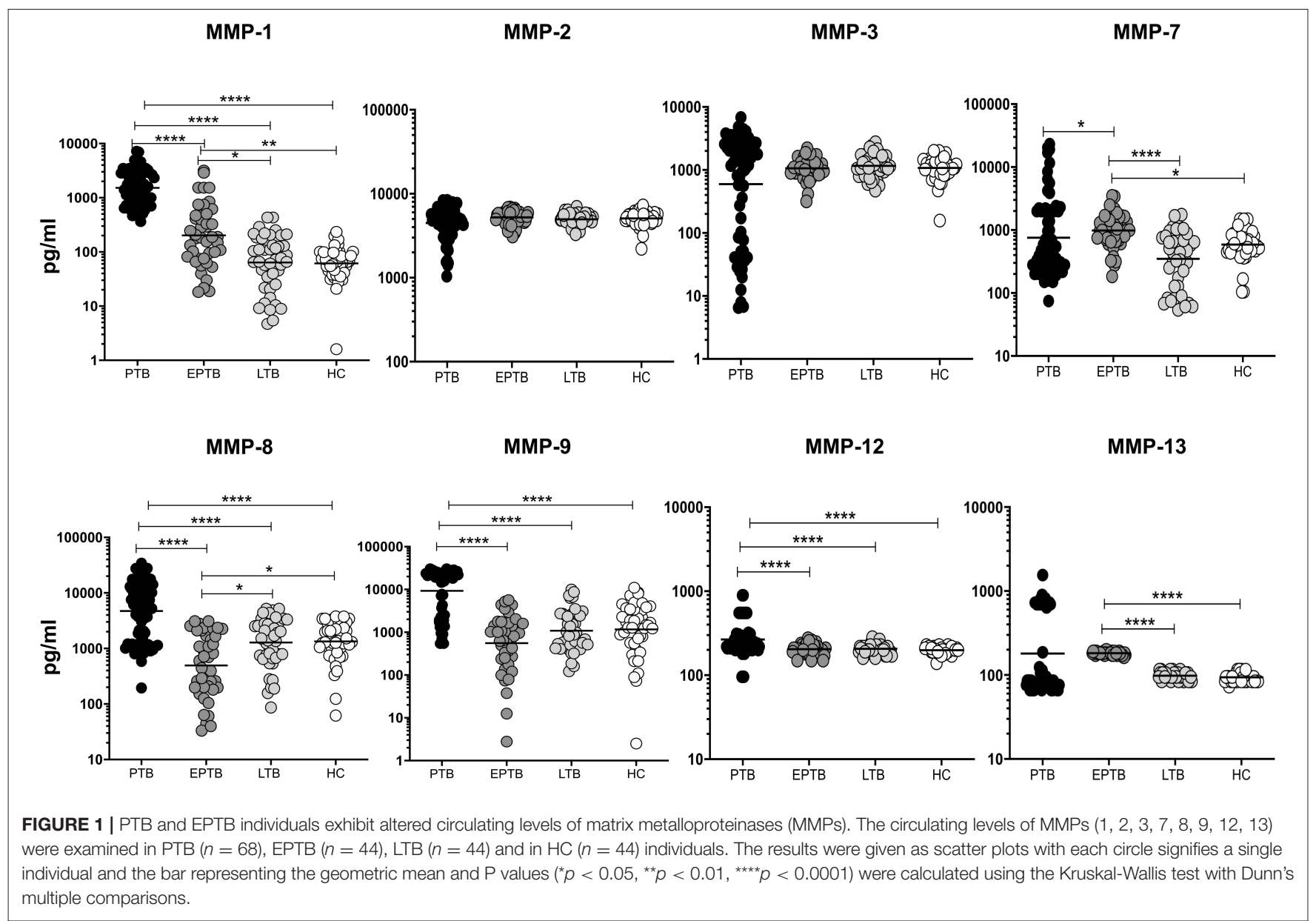

comparison with LTB and HC individuals (Figure 2). Hence, both PTB and EPTB are associated with altered circulating levels of TIMPs.

\section{Post-treatment Modulation of MMPs in PTB Individuals}

We measured the pre and post-treatment circulating levels of MMPs in a subset of PTB individuals (Figure 3). As shown in Figure 3, the systemic levels of MMP-7 [3855.0 pg/ml in BL vs. $7653.0 \mathrm{pg} / \mathrm{ml}$ in post-T], MMP-8 [1833.0 pg/ml in BL vs. 3734.0 $\mathrm{pg} / \mathrm{ml}$ in post-T], MMP-9 [1849.0 pg/ml in BL vs. $3060.0 \mathrm{pg} / \mathrm{ml}$ in post-T], MMP-12 [353.0 pg/ml in BL vs. $964.2 \mathrm{pg} / \mathrm{ml}$ in post$\mathrm{T}]$ and MMP-13 [765.8 pg/ml in BL vs. $975.1 \mathrm{pg} / \mathrm{ml}$ in post$\mathrm{T}$ ] were significantly increased in post-treatment condition than with pre-treatment levels among PTB individuals. However, the other MMPs $(1,2,3)$ were not significantly different between the two time points. Thus, PTB individuals are associated with increase of certain MMPs after ATT.

\section{Post-treatment Modulation of MMPs in EPTB Individuals}

To study the effect of ATT, we have measured the baseline (BL) and post-treatment (post-T) systemic levels of MMPs (MMP-1, 2,
3, 7, 8, 9, 12, and 13) in EPTB individuals (Figure 4). As we shown in Figure 4, the circulating levels of MMPs were significantly (MMP-1 [146.2 pg/ml in BL vs. $87.82 \mathrm{pg} / \mathrm{ml}$ in post-T] and MMP7 [987.0 pg/ml in BL vs. $87.82 \mathrm{pg} / \mathrm{ml}$ in post-T] was diminished) and (MMP-8 [575.5 pg/ml in BL vs. $1052.0 \mathrm{pg} / \mathrm{ml}$ in post-T] and MMP-9 [558.0 pg/ml in BL vs. $1512.0 \mathrm{pg} / \mathrm{ml}$ in post-T] was increased) altered between baseline and post-treatment condition of EPTB individuals. In contrast, the circulating levels of other MMPs (MMP-2, 3, 12, and 13) were not significantly modulated upon the completion of ATT.

\section{Post-treatment Modulation of TIMPs in PTB and EPTB Individuals}

Like MMPs, we also wanted to study the effect of ATT in TIMPs and examined the pre and post-treatment systemic levels of TIMP-1, 2, 3, 4 in PTB and EPTB individuals (Figure 5). As shown in Figure 5A, in PTB individuals, the circulating levels of TIMP-1 $(28440.0 \mathrm{pg} / \mathrm{ml}$ in BL vs. $254551.0 \mathrm{pg} / \mathrm{ml}$ in postT) and TIMP-4 (2188.0 pg/ml in BL vs. $4364.0 \mathrm{pg} / \mathrm{ml}$ in post$\mathrm{T})$ were significantly increased at post-treatment compared to pre-treatment levels. In contrast, the systemic levels of TIMP$2(5546.0 \mathrm{pg} / \mathrm{ml}$ in BL vs. $2107.0 \mathrm{pg} / \mathrm{ml}$ in post-T) and TIMP-3 (1141.0 pg/ml in BL vs. $555.5 \mathrm{pg} / \mathrm{ml}$ in post-T) were significantly 
TIMP-1

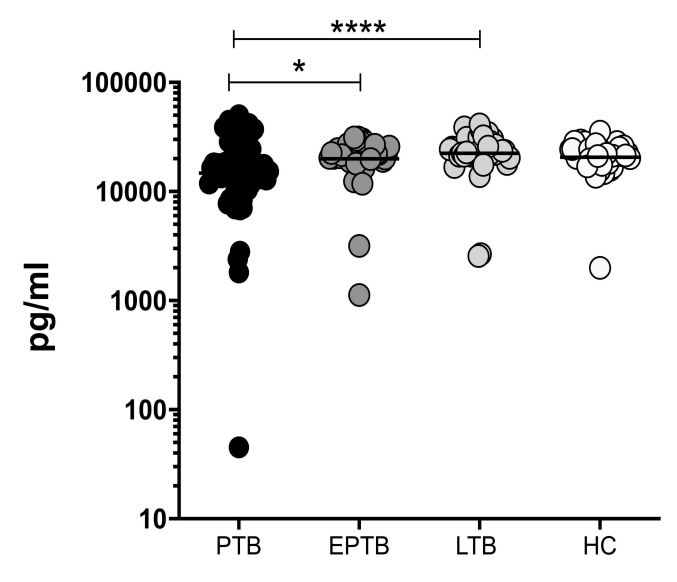

TIMP-3

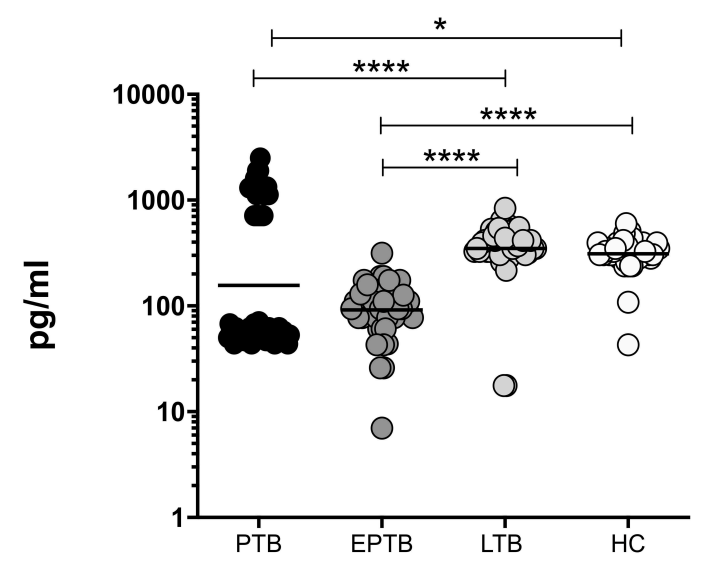

TIMP-2

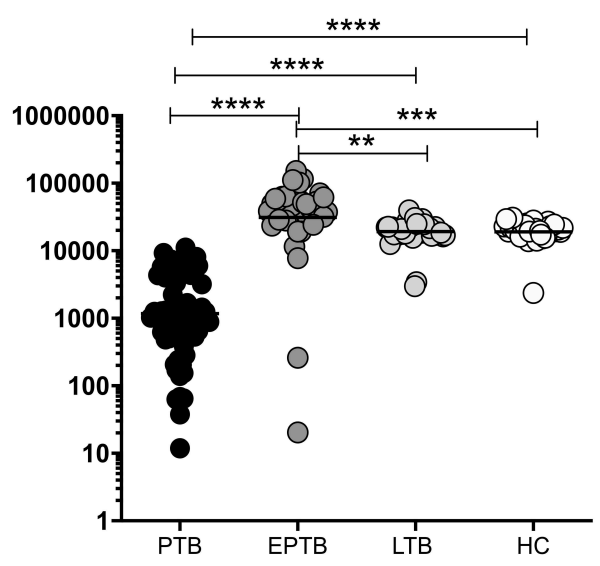

TIMP-4

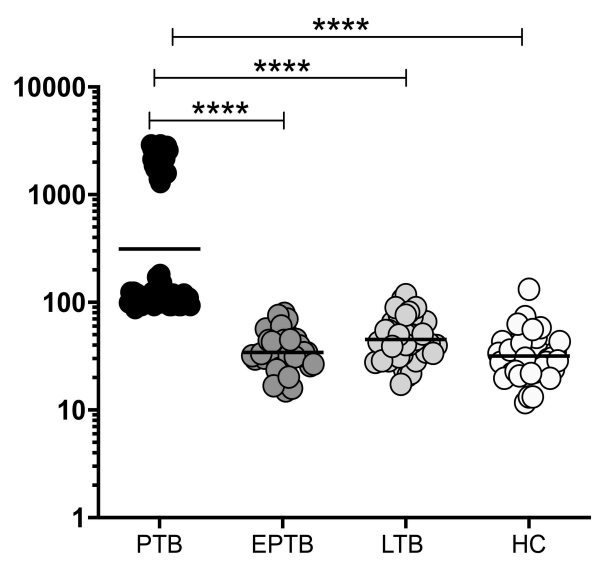

FIGURE 2 | PTB and EPTB individuals are associated with altered plasma levels of TIMPs. The systemic levels of TIMPs $(1,2,3,4)$ were examined in PTB $(n=68)$, EPTB $(n=44)$, LTB $(n=44)$, and in HC $(n=44)$ individuals. The results were given as scatter plots with each circle indicates a single individual and the bar representing the geometric mean. P values $\left({ }^{*} p<0.05,{ }^{* *} p<0.01,{ }^{* \star *} p<0.001,{ }^{\star \star \star *} p<0.0001\right)$ were calculated using the Kruskal-Wallis test with Dunn's multiple comparisons.

diminished at post-treatment compared to pre-treatment levels (Figure 5A). The circulating levels of TIMP-4 (34.19 pg/ml in BL vs. $28.42 \mathrm{pg} / \mathrm{ml}$ in post-T) was significantly decreased between baseline and post-treatment condition of EPTB individuals. In contrast, the systemic levels of TIMP-1, 2, 3 were not significantly altered between the baseline and post-treatment condition of EPTB individuals (Figure 5B).

\section{MMPs $(1,9)$ and TIMPs $(2,4)$ Distinguish PTB From EPTB, LTB, and/or HC Individuals}

To elucidate whether MMPs and TIMPs can distinguish PTB from other groups, we performed ROC analysis (Table 2). Among the MMPs analyzed, MMP-1 (PTB vs. EPTB-sensitivity95.59, specificity-84.09, AUC-0.9418 and $P<0.0001$; PTB vs. LTB-sensitivity-98.53, specificity-100, AUC-0.9993 and $P<0.0001$; PTB vs. HC-sensitivity-100, specificity-100, AUC-1 and $P<0.0001$ ) and MMP-9 (PTB vs. EPTB-sensitivity-80.88, specificity-84.09, AUC-0.9094 and $P<0.0001$; PTB vs. LTBsensitivity-73.53, specificity-75.00, AUC-0.8763, and $P<0.0001$; PTB vs. HC-sensitivity-100, specificity-100, AUC-1 and $P<0.0001)$ could significantly discriminate between the study individuals. Similarly, TIMP-2 (PTB vs. EPTB-sensitivity-95.59, specificity-95.45, AUC-0.9576 and $P<0.0001$; PTB vs. LTBsensitivity-100, specificity-95.45, AUC-0.9853 and $P<0.0001$; PTB vs. HC-sensitivity-100, specificity-97.73, AUC-0.9923, and $P<0.0001$ ) and TIMP-4 (PTB vs. EPTB-sensitivity-100, specificity-100, AUC-1 and $P<0.0001$; PTB vs. LTB-sensitivity98.53, specificity-95.45, AUC-0.9789 and $P<0.0001$; PTB vs. HC-sensitivity-100, specificity-97.73, AUC-0.9866 and $P<0.0001)$ could significantly distinguish PTB from other 


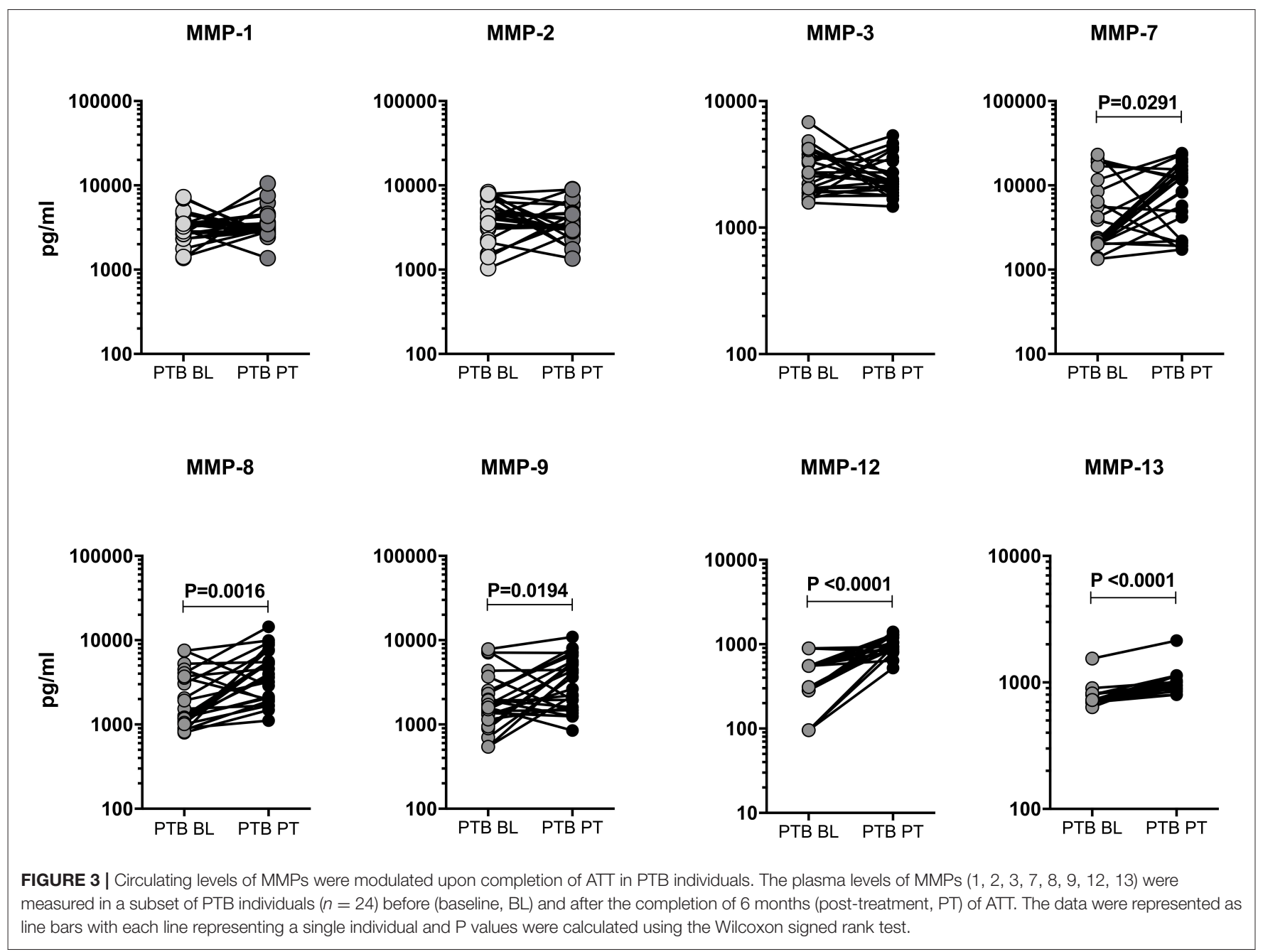

study groups (Table 2). Hence, MMP-1, 9 and TIMP-2, 4 are potential markers in distinguishing PTB from EPTB, LTB, and HC groups.

\section{MMP-13 and TIMP-2 Discriminate EPTB From LTB and HC Individuals}

Similar to PTB, the ROC analysis of MMPs and TIMPs was carried out between EPTB, LTB, and HC individuals (Table 3). Among the MMPs analyzed, MMP-13 (EPTB vs. LTB-sensitivity-100, specificity-100, AUC-1, and $P<0.0001$; EPTB vs. HC-sensitivity-100, specificity-100, AUC-1 and $P<0.0001)$ could significantly discriminate EPTB from LTB and HC individuals. As shown in Table 3, the systemic levels of TIMP-2 could significantly distinguish EPTB from LTB (sensitivity-81.82, specificity-90.91, AUC-0.8631, and $P<0.0001$ ) and HC (sensitivity-84.09, specificity-84.09, AUC0.8719 , and $P<0.0001$ ) individuals. Thus, MMP-13 and TIMP-2 are potential candidate markers for distinguishing EPTB from LTB and HC individuals.

\section{Relationship Between MMPs/TIMPs in Different TB Infected and HC Individuals}

We next examined the correlation of MMPs $(1,2,3,7,8,9,12,13)$ with TIMPs $(1,2,3,4)$ in the different group of (PTB, EPTB, LTB, and HC) individuals. As shown in Figure 6, MMP-2 and MMP3 were positively correlated and MMP- 8 was negative correlated with all TIMPs $(P<0.0001)$; whereas, a significant positive correlation was observed for MMP-1, MMP-7, and MMP-13 with TIMP-1 and/or TIMP-4 in PTB individuals. In contrast, MMP-12 exhibited a significant negative correlation with TIMP2 and TIMP-3 in PTB individuals (Figure 6A). Similarly, MMP-1 and MMP-3 were positively correlated with TIMP-1 and TIMP-2, whereas MMP-9 and MMP-13 were positively correlated with TIMP-2 alone in EPTB individuals (Figure 6B). As shown in Figure 6C, MMP-3 levels were positively correlated with all TIMPs; whereas, MMP-2, MMP-8, and MMP-12 were positive correlated with TIMP-4/TIMP-3/TIMP-1 and TIMP4, respectively, in LTB individuals. Finally, the plasma levels of MMP-8 and MMP-13 were shown to be positively correlated with certain TIMPs $(2,3,4)$ and $(1,2)$ in HC individuals (Figure 6D). 


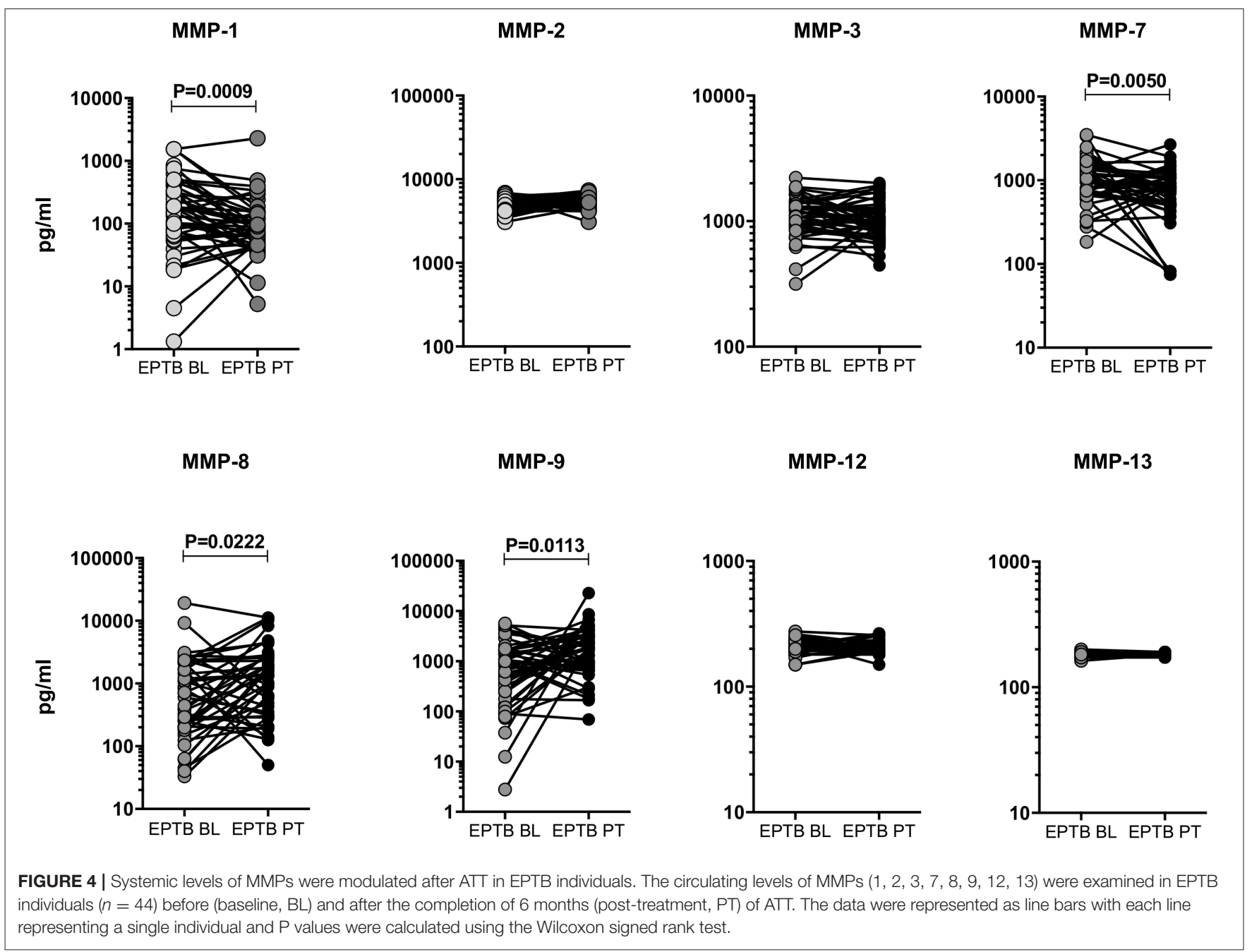

Thus, based on our observation certain MMPs appears to be significantly correlated with TIMPs.

\section{PCA Analysis of MMPs/TIMPs Between the Study Individuals}

Next, we analyzed the impact of MMPs $(1,2,3,7,8,9,12$, 13) and TIMPs $(1,2,3,4)$ in discriminating the diseased individuals from infected and uninfected individuals by PCA analysis. We have utilized their plasma levels to generate the clusters for PTB and EPTB individuals and compared them with LTB and HC individuals (Figure 7). We observed distinct clustering of MMPs/TIMPs for both PTB (component 1-43.1\% and component 2-24.6) and EPTB individuals (component 1-26.4\% and component 2-22.2) which are able to clearly discriminate from LTB and HC individuals (Figures 7A,B).

\section{Logistic Regression Analysis of PTB and EPTB}

Finally, we have performed univariate and multivariate analysis (95\% confidence interval [CI]) of MMPs $(1,2,3,7,8,9,12,13$ ) and TIMPs $(1,2,3,4)$ in both PTB and EPTB after the adjustment for confounding factors like age and gender to identify whether they might possibly serve as biomarkers for PTB and EPTB disease. As shown in Table 4, we found that by both univariate and multivariate analysis, MMP-1, 7, 8, 9, 12, and 13 were associated with significantly greater odds of PTB, while MMP-2 and 3 were associated with significantly decreased odd of PTB. Similarly, TIMP-4 was associated with highly elevated odd of PTB, while TIMP- 1 and 2 were associated with decreased odds of PTB. As shown in Table 5, we also found that by both univariate and multivariate analysis, MMP-2, TIMP-1, 2, 3 were associated with significantly greater odds of EPTB while MMP-1, 7, 9, 13 and TIMP-4 were associated with significantly decreased odd of EPTB. Thus, logistic regression analysis identified the odds ratio of MMP and TIMP levels being associated with increased or decreased likelihood of having PTB or EPTB.

\section{DISCUSSION}

The identification of definitive non-sputum, blood-based biomarkers for PTB and ЕРTB is essential either for disease diagnosis or for chemotherapy treatment monitoring. It is also 


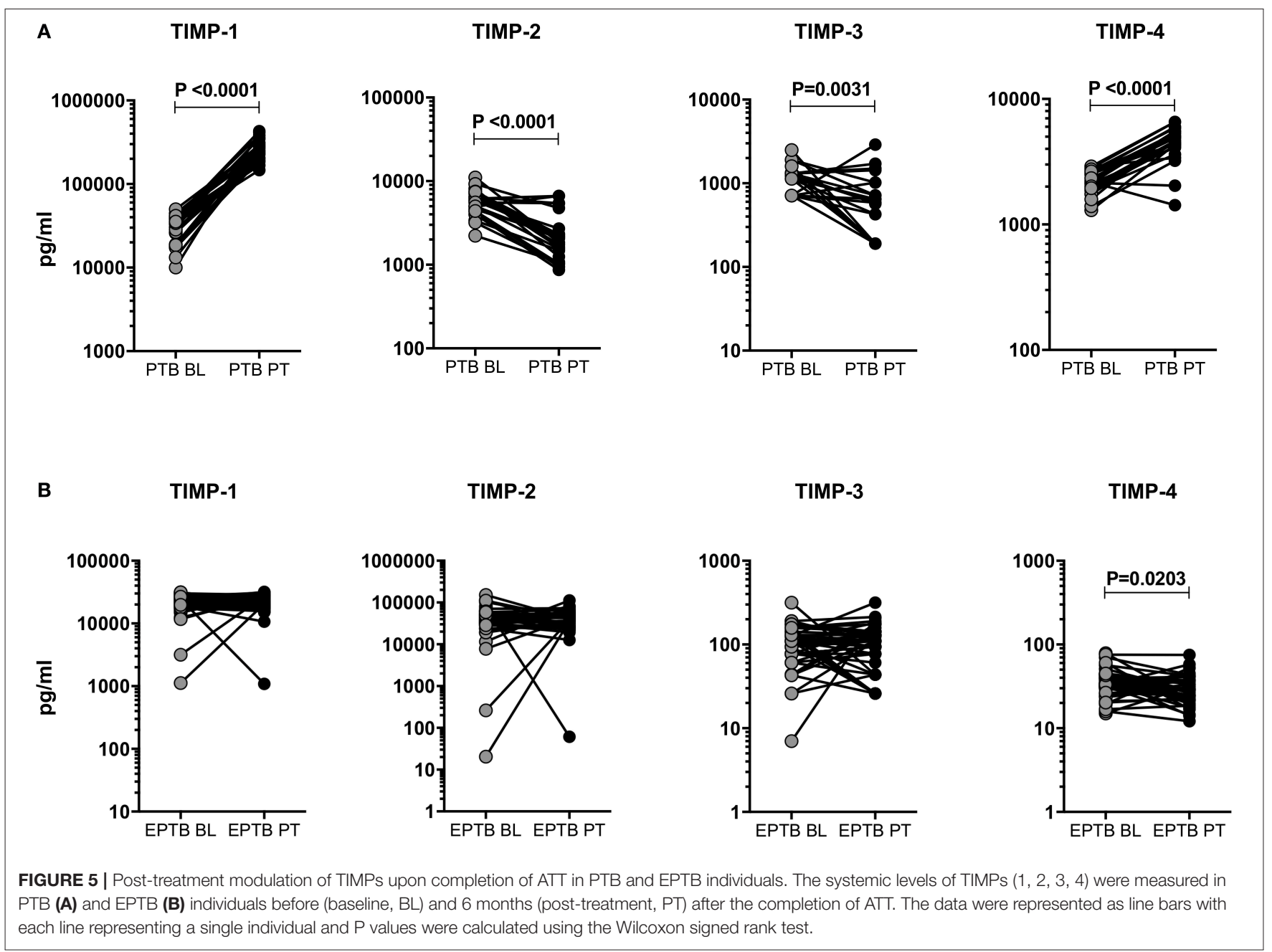

TABLE 1 | Demographics of the study individuals.

\begin{tabular}{lcccc}
\hline Study demographics & PTB & EPTB & LTB & HC \\
\hline $\begin{array}{l}\text { Number of subjects } \\
\text { recruited }(n)\end{array}$ & 68 & 44 & 44 & 44 \\
$\begin{array}{l}\text { Gender (M/F) } \\
\text { Median age in years }\end{array}$ & $41(19-54)$ & $30(18-51)$ & $32(21-62)$ & $34(21-55)$ \\
$\begin{array}{l}\text { (Range) } \\
\begin{array}{l}\text { Culture/smear grade } \\
\text { (0/1+/2+/3+) }\end{array}\end{array}$ & $0 / 23 / 27 / 18$ & $8 / 34 / 2 / 0$ & Not done & Not done \\
QuantiFERON-TB Gold & Not done & Not done & Positive & Negative \\
\hline
\end{tabular}

worthwhile to understand the architecture and mechanism of disease pathogenesis which might provide additional targets for immune mediated therapies. The role of certain MMPs and TIMPs were studied previously in the immunopathology of pulmonary $\mathrm{TB}$ infection and certain forms of EPTB. Nevertheless, a comprehensive examination of a complete panel of MMPs and TIMPs in PTB and EPTB has not been performed till now. Hence in the present study, we have examined the association of MMPs and TIMPs in two different forms of TB and compared them with LTB and HCs. The systemic levels of both MMPs and TIMPs disclose that an alteration occurred between the PTB and EPTB patients indicating their disease presentation profile differs significantly. ROC analysis discriminated between the diseased and the other study groups among some of the MMPs and TIMPs suggesting that they have the potential ability to be used as a blood-based biomarker.

Several studies have revealed that PTB individuals are characterized by enhanced MMPs (MMP-1, 2, 3, 7, 8, 9) in sputum, pleural fluid and bronchoalveolar lavage (BAL) fluids $(17,22-25)$. Our data were also consistent with the above findings and we show that the circulating levels of MMPs (1, $8,9,12)$ and MMPs $(1,7,13)$ were significantly increased in both PTB and EPTB individuals. Enhanced systemic levels of MMP-1 in both TB diseased groups supports the premise that it is an absolute indicator of tissue matrix damage, higher alveolar destruction and breakdown of collagen. Another study has also revealed that MMP-1 triggers the lung matrix destruction and their levels were increased compared to latently infected 
TABLE 2 | MMPs (1, 9) and TIMPs (2, 4) clearly distinguish PTB from EPTB, LTB, and/or HC individuals.

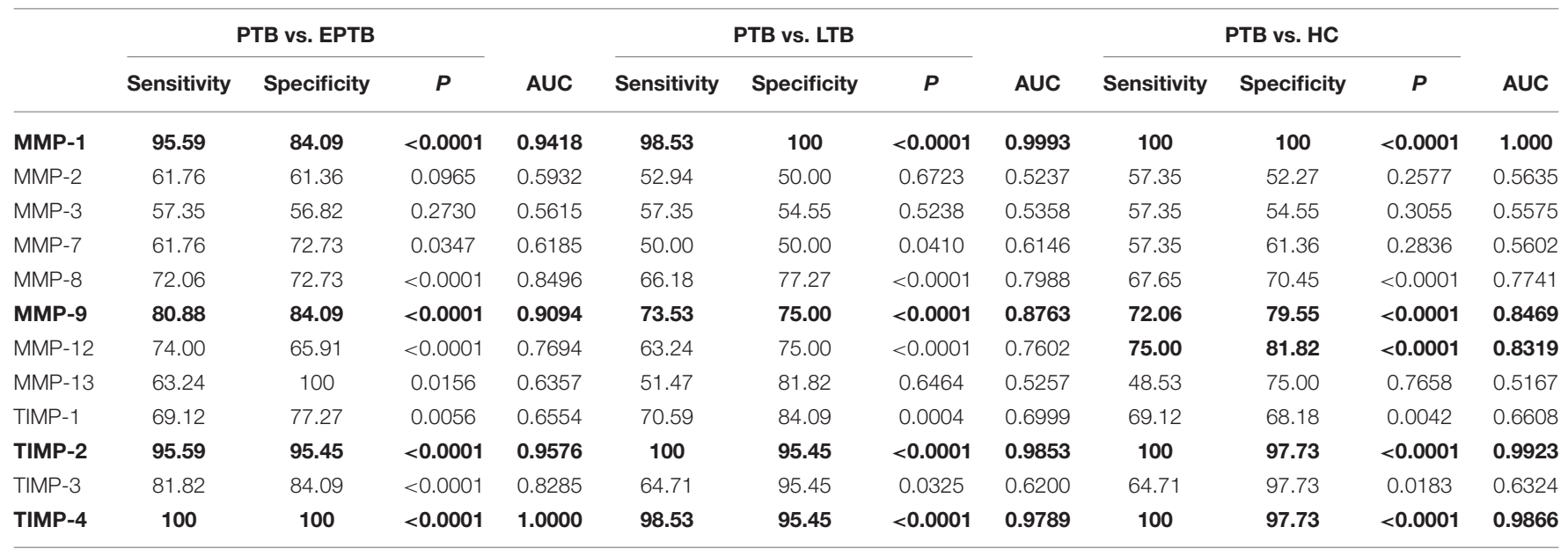

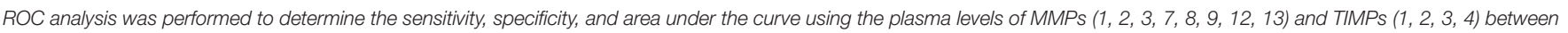

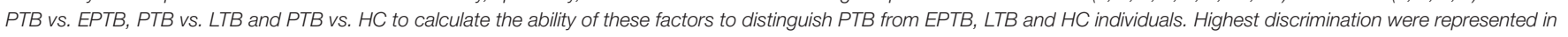
bold.

TABLE 3 | MMP-13 and TIMP-2 clearly distinguishes EPTB from LTB and HC individuals.

\begin{tabular}{|c|c|c|c|c|c|c|c|c|}
\hline & \multicolumn{3}{|c|}{ EPTB vs. LTB } & AUC & \multicolumn{3}{|c|}{ EPTB vs. HC } & AUC \\
\hline MMP-2 & 61.36 & 65.19 & 0.0489 & 0.6219 & 59.09 & 54.55 & 0.5579 & 0.3500 \\
\hline MMP-3 & 56.82 & 54.55 & 0.3247 & 0.5610 & 54.55 & 52.77 & 0.7011 & 0.5238 \\
\hline MMP-7 & 75.00 & 70.45 & $<0.0001$ & 0.8011 & 75.00 & 61.36 & 0.0003 & 0.7237 \\
\hline MMP-12 & 52.27 & 54.55 & 0.9800 & 0.5015 & 52.27 & 61.36 & 0.1465 & 0.5889 \\
\hline MMP-13 & 100 & 100 & $<0.0001$ & 1 & 100 & 100 & $<0.0001$ & 1 \\
\hline TIMP-1 & 56.82 & 63.64 & 0.0639 & 0.6147 & 59.09 & 52.27 & 0.4678 & 0.5449 \\
\hline TIMP-2 & 81.82 & 90.91 & $<0.0001$ & 0.8631 & 84.09 & 84.09 & $<0.0001$ & 0.8719 \\
\hline TIMP-3 & 64.71 & 81.82 & 0.0344 & 0.6186 & 97.73 & 95.45 & $<0.0001$ & 0.9587 \\
\hline
\end{tabular}

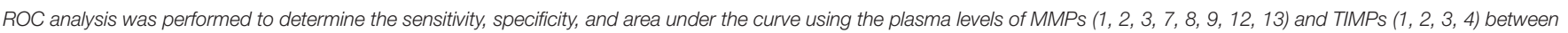
EPTB vs. LTB and EPTB vs. HC individuals to calculate the ability of these factors to distinguish EPTB from LTB and HC individuals. Highest discrimination were represented in bold.

individuals (26). Even microarray profiling has shown increased (660 fold) MMP-1 gene expression in human TB granulomas, cavity areas (rabbit model) and macaque lungs compared to healthy lungs (27-29). Previous study also highlighted similar data indicating elevated MMP-1 and HO-1 levels were highly discriminatory between active TB and LTB individuals (30). It has been observed that increased levels of MMPs (2, 8, 9) at diagnosis and higher MMP-3 and 8 at 2 weeks were connected to culture positivity in sputum samples at 2-weeks of infection. After the first 6 weeks of treatment initiation, both MMP-1 and 8 levels remains high with delayed sputum culture conversion (31). We also describe similar post-treatment data on MMP-8 where the circulating levels are higher after treatment and MMP-1 levels were not significantly altered after chemotherapy.
In addition, systemic levels of MMPs $(1,7,8)$ were significantly increased in children with active TB than healthy individuals (32). Elevated sputum and plasma levels of MMP8 are present in TB individuals, TB- immune reconstitution inflammatory syndrome (IRIS) and in individuals with and without HIV co-infection (33-35). MMP-9 levels were correlated with disease severity and increases the susceptibility TB infection (36). The levels of MMP-9 were elevated in the cerebrospinal fluid of TB meningitis patients and pleural fluid. Elevated levels of MMP-12 were observed in the COPD patient than the control groups (37). Higher secretion of MMP-9 as found upon Mtb infection of both monocytes and macrophages, and was shown to be important for granuloma formation (38, 39). It also been shown that MMP-9 gene knockout mice have poor granuloma architecture and reduced recruitment 
A

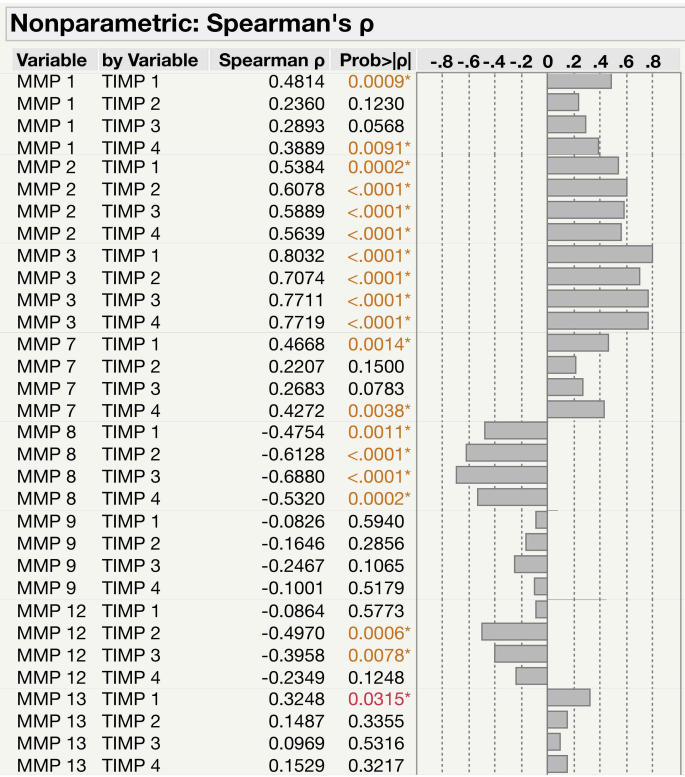

C

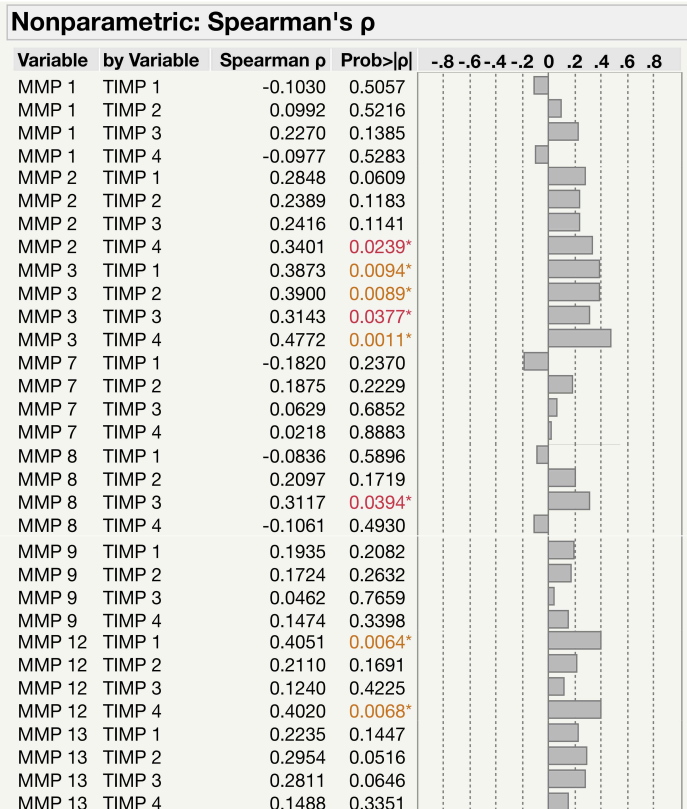

B

\begin{tabular}{|c|c|c|c|c|c|c|}
\hline \multicolumn{7}{|c|}{ Nonparametric: Spearman's $\rho$} \\
\hline Variable & by Variable & Spearman $\rho$ & Prob $>|\rho|$ & $-.8-.6-.4-.2$ & 0.2. & .6 .8 \\
\hline MMP 1 & TIMP 1 & 0.4099 & $0.0057^{*}$ & & & \\
\hline MMP 1 & TIMP 2 & 0.3414 & $0.0233^{\star}$ & & & \\
\hline MMP 1 & TIMP 3 & 0.2427 & 0.1125 & & & \\
\hline MMP 1 & TIMP 4 & 0.5457 & $0.0001^{*}$ & & & \\
\hline MMP 2 & TIMP 1 & 0.2366 & 0.1220 & & & \\
\hline MMP 2 & TIMP 2 & 0.2639 & 0.0834 & & & \\
\hline MMP 2 & TIMP 3 & -0.0382 & 0.8056 & & & \\
\hline MMP 2 & TIMP 4 & 0.1264 & 0.4135 & & & \\
\hline MMP 3 & TIMP 1 & 0.3614 & $0.0159^{*}$ & & & \\
\hline MMP 3 & TIMP 2 & 0.3577 & $0.0171^{*}$ & & & \\
\hline MMP 3 & TIMP 3 & 0.1113 & 0.4720 & & & \\
\hline MMP 3 & TIMP 4 & $\begin{array}{r}0.2453 \\
-0.0468\end{array}$ & 0.1085 & & & \\
\hline MMP 7 & TIMP 2 & 0.1868 & 0.2246 & & & \\
\hline MMP 7 & TIMP 3 & -0.1231 & 0.4261 & & & \\
\hline MMP 7 & TIMP 4 & 0.0804 & 0.6037 & & & \\
\hline MMP 8 & TIMP 1 & 0.0734 & 0.6357 & & & \\
\hline MMP 8 & TIMP 2 & 0.1236 & 0.4241 & & & \\
\hline MMP 8 & TIMP 3 & 0.1290 & 0.4039 & & & \\
\hline MMP 8 & TIMP 4 & 0.1014 & 0.5125 & & & \\
\hline MMP 9 & TIMP 1 & 0.1810 & 0.2397 & & & \\
\hline MMP 9 & TIMP 2 & 0.3863 & $0.0096^{*}$ & & & \\
\hline MMP 9 & TIMP 3 & 0.0420 & 0.7866 & & & \\
\hline MMP 9 & TIMP 4 & 0.0269 & 0.8622 & & & \\
\hline MMP 12 & TIMP 1 & 0.1609 & 0.2966 & & & \\
\hline MMP 12 & TIMP 2 & 0.2959 & 0.0512 & & & \\
\hline MMP 12 & TIMP 3 & 0.0873 & 0.5730 & & & \\
\hline MMP 12 & TIMP 4 & 0.2914 & 0.0550 & & & \\
\hline MMP 13 & TIMP 1 & 0.2802 & 0.0655 & & & \\
\hline MMP 13 & TIMP 2 & 0.3407 & $0.0236^{*}$ & & & \\
\hline MMP 13 & TIMP 3 & -0.0818 & 0.5975 & & & \\
\hline & & & 0.0681 & & & \\
\hline
\end{tabular}

D

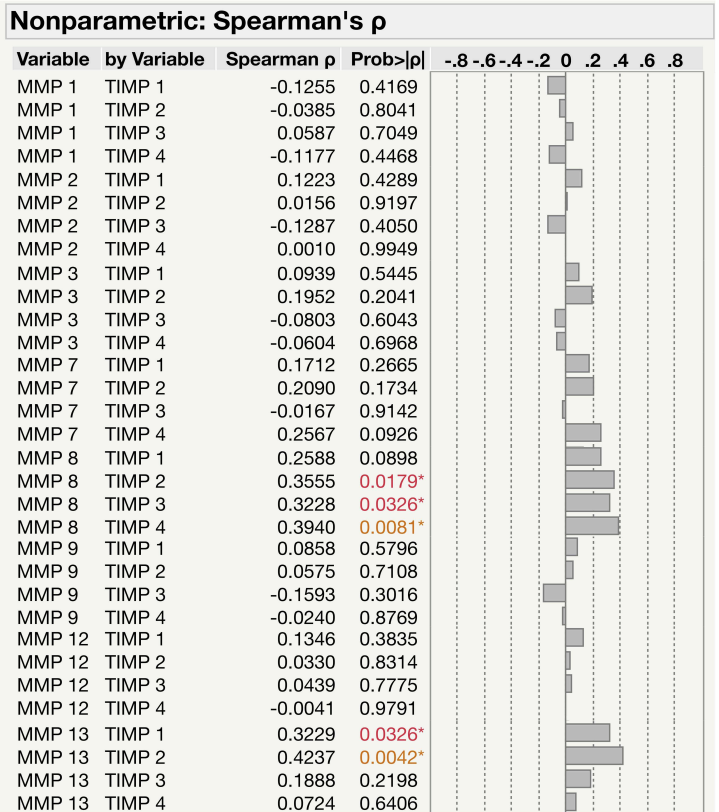

FIGURE 6 | Correlation between the MMPs (1, 2, 3, 7, 8, 9, 12, 13) and TIMPs (1, 2, 3, 4) levels among the study individuals (A) PTB (B) EPTB (C) LTB and (D) HCs. The data were represented as table and the significant differences were given in orange or red color.

of macrophages (36). Similarly, increased MMP-8 levels in PTB but not EPTB implies that this neutrophil-derived MMP could be related to severe form of TB disease (40). Likewise, our data were similar to the above findings by showing increased levels of MMP-8, MMP-9, and MMP-12 in PTB compared EPTB, LTB and HC groups. Their increased levels might be either deleterious to the host or important for the maintenance of the active granuloma. Even the post treatment systemic levels of MMP-9, 12, 13 were higher compared to pre-treatment indicating MMP levels could serve as additional biomarkers for successful chemotherapy. In addition, the ROC analysis revealed certain MMPs $(1,9)$ and MMP-12 (PTB vs. HC) were potentially capable of being a good peripheral bio-markers for PTB to separate from other diseased or control individuals.

In contrast, MMP-8 were significantly diminished in EPTB compared to LTB and HC groups. The reason could be because of different site of infection between the two TB infected groups. Hence, both diseased groups differ significantly on their expression levels stating that it might be used as a 
A

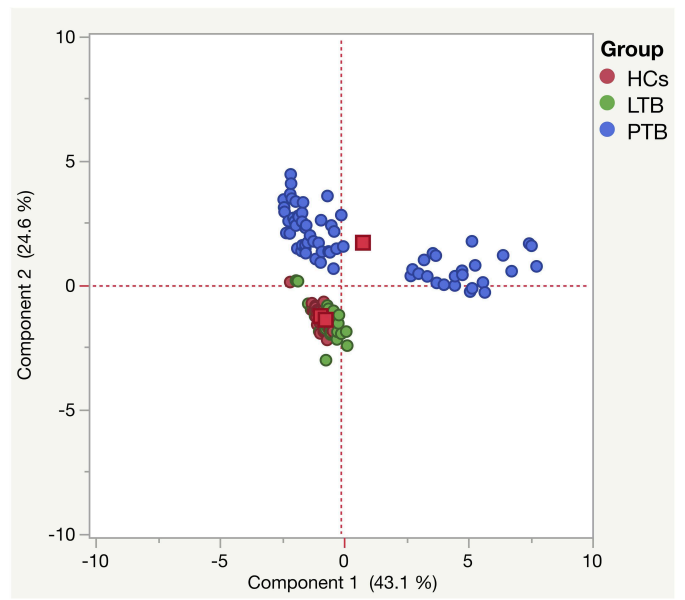

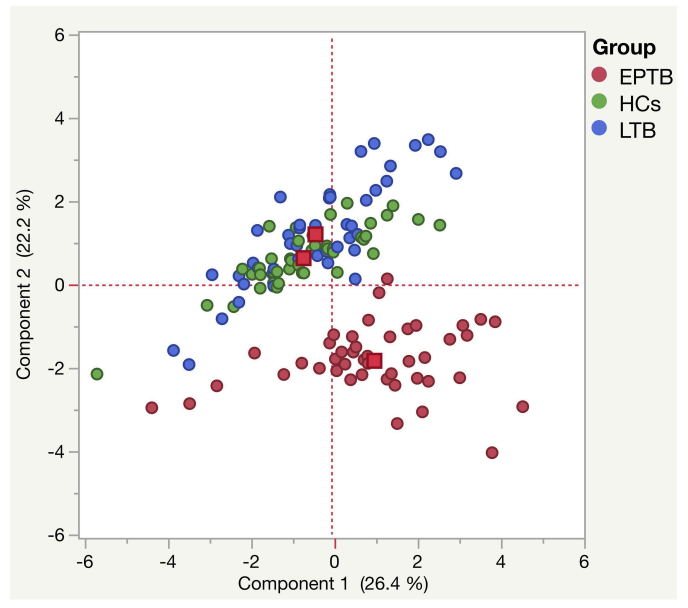

FIGURE 7 | Principle component analysis (PCA) plots of MMPs (1, 2, 3, 7, 8, 9, 12, 13) and TIMPs (1, 2, 3, 4) (A) PTB (blue) vs. LTB (green) vs. HC (red) and (B) EPTB (red) vs. LTB (blue) vs. HC (green) individuals. PCA plot shows the ELISA data from the combination of three different experimental groups.

TABLE 4 | Logistic regression model to identify the biomarkers for PTB.

\begin{tabular}{|c|c|c|c|c|}
\hline & \multicolumn{2}{|l|}{ Univariate } & \multicolumn{2}{|l|}{ Multivariate } \\
\hline & OR (95\% Cl) & $P$ & aOR $(95 \% \mathrm{Cl})^{*}$ & $P$ \\
\hline MMP1 & $9.15(4.28-19.56)$ & $<0.001$ & $10.34(3.31-32.27)$ & $<0.001$ \\
\hline MMP2 & $0.64(0.52-0.77)$ & $<0.001$ & $0.65(0.53-0.79)$ & $<0.001$ \\
\hline MMP3 & $0.76(0.64-0.9)$ & 0.002 & $0.75(0.62-0.92)$ & 0.005 \\
\hline MMP7 & $19.7(7.93-48.9)$ & $<0.001$ & $37.73(11.25-126.58)$ & $<0.001$ \\
\hline MMP8 & $2.25(1.75-2.89)$ & $<0.001$ & $2.07(1.61-2.67)$ & $<0.001$ \\
\hline MMP9 & $2.46(1.94-3.13)$ & $<0.001$ & $2.35(1.84-3.01)$ & $<0.001$ \\
\hline MMP12 & $26.53(6.54-107.61)$ & $<0.001$ & 28.54 (6.75-120.64) & $<0.001$ \\
\hline MMP13 & $1.74(1.3-2.34)$ & $<0.001$ & 2.04 (1.44-2.9) & $<0.001$ \\
\hline TIMP1 & $0.58(0.4-0.83)$ & 0.003 & $0.58(0.4-0.84)$ & 0.004 \\
\hline TIMP2 & $0.25(0.16-0.37)$ & $<0.001$ & $0.25(0.16-0.38)$ & $<0.001$ \\
\hline TIMP3 & $0.84(0.69-1.01)$ & 0.057 & $0.85(0.7-1.03)$ & 0.096 \\
\hline TIMP4 & $3022.8(65.04-140484.7)$ & $<0.001$ & $1164.4(43.34-31287.9)$ & $<0.001$ \\
\hline
\end{tabular}

*Multivariate model was adjusted for age and gender.

peripheral based diagnostic bio-marker for EPTB disease. After the completion of ATT, the systemic levels of MMP-8 were significantly downregulated in EPTB individuals. Our data also shows higher circulating levels of MMP-7 and MMP-13 in EPTB individuals compared to PTB, LTB and/or HC individuals. The post-treatment circulating levels of MMP-7 was significantly decreased compared to pre-treatment levels. MMP-13 could potentially be of use as a blood-based bio-marker for diagnosis of EPTB disease. Consistent with our data, the mRNA expression levels of certain MMPs $(1,3,12,13)$ were highly upregulated in macrophages or epithelial tissues isolated from infected tissues (41).

It has been implied that Mtb dynamically impairs the equilibrium between MMPs and TIMPs. Moreover, whether
TABLE 5 | Logistic regression model to identify the biomarkers for EPTB.

\begin{tabular}{|c|c|c|c|c|}
\hline & \multicolumn{2}{|c|}{ Univariate } & \multicolumn{2}{|c|}{ Multivariate } \\
\hline & OR (95\% Cl) & $P$ & aOR $(95 \% \mathrm{Cl})^{*}$ & $\boldsymbol{P}$ \\
\hline MMP1 & $0.66(0.55-0.78)$ & $<0.001$ & $0.62(0.52-0.75)$ & $<0.001$ \\
\hline MMP2 & $1.26(1.04-1.52)$ & 0.020 & $1.26(1.04-1.53)$ & 0.017 \\
\hline MMP3 & $1.22(0.95-1.55)$ & 0.115 & $1.21(0.95-1.54)$ & 0.125 \\
\hline MMP7 & $0.31(0.21-0.45)$ & $<0.001$ & $0.29(0.19-0.44)$ & $<0.001$ \\
\hline MMP8 & $0.87(0.74-1.02)$ & 0.084 & $0.84(0.7-1)$ & 0.052 \\
\hline MMP9 & $0.85(0.74-0.97)$ & 0.014 & $0.81(0.7-0.94)$ & 0.005 \\
\hline MMP12 & $0.39(0.14-1.06)$ & 0.064 & $0.37(0.13-1.01)$ & 0.053 \\
\hline MMP13 & $0.33(0.17-0.66)$ & 0.002 & $0.33(0.16-0.68)$ & 0.003 \\
\hline TIMP1 & $1.78(1.02-3.13)$ & 0.044 & $1.78(1.01-3.14)$ & 0.045 \\
\hline TIMP2 & $1.41(1.15-1.73)$ & 0.001 & $1.6(1.24-2.06)$ & $<0.001$ \\
\hline TIMP3 & $1.6(1.26-2.03)$ & $<0.001$ & $1.58(1.24-2.02)$ & $<0.001$ \\
\hline TIMP4 & 0.69 (0.53-0.91) & 0.008 & $0.65(0.49-0.88)$ & 0.005 \\
\hline
\end{tabular}

*Multivariate model was adjusted for age and gender.

TIMPs could be used as a potential immune based biomarker in PTB and EPTB remains unclear (29). In our study, the systemic levels of TIMP-1 were significantly reduced in both TB diseased groups. Similar to our observation, lower TIMP1 levels were reported in pulmonary secretions of TB patients (42). It has also been shown in cell culture experiments, the elevated levels of MMP is independent and not balanced by a higher TIMP-1 level (43). We also observed circulating TIMP-1 levels were decreased in active TB patients but not in ЕРTB individuals. We predict that lower TIMP-1 levels in PTB could be due to unrestricted gelatinolytic action within the granuloma architecture with a subsequent propensity for matrix degradation. ROC analysis of TIMP-1 potentially discriminated PTB from EPTB and other control groups. Hence, we propose 
that TIMP-1 could be a better biomarker for active TB diagnosis alone.

Similar to TIMP-1, TIMP-2 plasma levels were also significantly diminished in PTB and elevated in EPTB compared to LTB and HC individuals. Hence, it might be used as a potential biomarker for discriminating between PTB and EPTB disease and also from LTB and HC individuals. This is in contrast to some other studies where they displayed significantly higher TIMP-1 and TIMP-2 levels in TB cases compared to healthy controls $(17,42)$. The reason behind this difference observed between the various data are yet to be explored. We have also shown that the circulating levels of TIMP-3 were significantly decreased in both the diseased groups. Previous study has revealed that TIMP-3 was greatly decreased in in-vitro human monocyte infection models but not at the tissue site (43). There was also evidence from mice models showing the reduction in TIMP-3 levels was connected with degradation of extra cellular matrix (44). Finally, our data on TIMP-4 revealed an increase in PTB compared to EPTB, LTB and HC individuals. In PTB, the systemic levels of TIMPs (TIMP-1, 4 were increased and TIMP-2, 3 were diminished) were altered after the completion of chemotherapy. In contrast, TIMP-4 alone was significantly modulated after the treatment in EPTB individuals. Finally, it was clearly seen from the ROC data of TIMPs where PTB (TIMP-2 and TIMP-4) and EPTB (TIMP-2) were highly discriminated from LTB and HC individuals. Hence, the above mentioned TIMPs might be a very good blood-based biomarker for PTB and EPTB diagnosis.

Our PCA analysis reveals that MMPs and TIMPs as a whole are useful parameters to distinguish PTB from LTB and HC individuals and EPTB from LTB and HC individuals with minimal overlapping distributions. This greatly adds to the growing evidence in the literature about the importance of MMPs and TIMPs are potential biomarkers of PTB and EPTB. Moreover, our data also provide additional insight into the equilibrium between MMPs and TIMPs in the different study groups by comparing the correlation matrices of these biomarkers. While, we observe a mostly positive correlation in EPTB, we observe a mix of positive and negative associations in PTB with MMP/TIMP correlations. Finally, our data also reveal the associations of MMPs and TIMPs with either increased or decreased risk of PTB and EPTB with certain MMPs and TIMPs being clearly positively associated and other being negatively associated with pulmonary or extrapulmonary disease. This is therefore an important value addition tool to the armamentarium of biomarkers reflecting these disease processes.

The limitations of the study include the moderate sample size, the lack of a validation cohort, the inclusion of only one form of EPTB and the absence of other bacterial, viral or parasitic infections. Overall from our observation, we suggest that differences in the systemic levels of various MMPs and TIMPs can be utilized as potential blood-based biomarkers for TB disease. Since, obtaining either sputum or bronchoalveolar lavage (BAL) fluids is challenging, this if validated in larger studies should provide a surrogate marker for these conditions.

\section{MATERIALS AND METHODS}

\section{Study Population}

The present study was approved by Institutional Review Board (NIRTIEC2010007) of National Institute for Research in Tuberculosis (NIRT), Chetpet, Chennai, Tamil Nadu, India and informed written consent form was acquired from all the study individuals. Our study consists of four different [PTB $(n=68)$, EPTB $(n=44)$, latent TB [LTB] $(n=$ $44)$, and healthy controls [HC] $(n=44)$ ] groups. The demographics of the study population are given in Table 1. PTB was diagnosed on the basis of culture positivity for Mtb by solid culture. EPTB diseased patients had only cervical lymphadenopathy and were diagnosed based on histopathology or bacteriological investigation comprising of GeneXpert or culture positive for Mtb. LTB individuals were positive for QuantiFERON TB-Gold (QFT) in tube assay and had lack of abnormalities in chest radiography and absence of any pulmonary symptoms. HCs were QFT negative and had lack of abnormalities in chest radiography and absence of any pulmonary symptoms. All the study individuals were HIV negative and devoid of steroid treatment and not affected with other chronic viral or bacterial infection. Plasma samples were collected at baseline (pre-treatment) from all the four groups of individuals. Both PTB and EPTB individuals were administered standard anti-tuberculosis treatment for 6 months and fresh plasma samples were collected from a subset of PTB $(n=24)$ and all EPTB $(n=44)$ individuals at the end of treatment.

\section{Immunoassays}

Plasma levels of human MMP-1, MMP-2, MMP-3, MMP7, MMP-8, MMP-9, MMP-12, MMP-13 (catalog number FCSTM07-8) and human TIMP-1, TIMP-2, TIMP-3, and TIMP4 (catalog number LKTM003) were measured using a Luminex kit purchased from R\&D Systems.

\section{Data Analysis}

The statistical significance between the various study (PTB, EPTB, LTB, and HC) population were analyzed using KruskalWallis test with Dunn's multiple comparisons. Wilcoxon signed rank test were used to measure the pre-and post-treatment systemic levels of MMPs and TIMPs. ROC analysis was used to measure the specificity and sensitivity between the study groups. GraphPad Prism version 8.0 (GraphPad Software Inc., San Diego, CA) were used to perform the statistical analysis and plotting the graphs. Both multivariate (Spearman rank correlation) and principal component analysis (PCA) (nonparametric) were performed using JMP (14.0 version). Finally, regression (univariate and multivariate) analysis were performed using STATA/MP version 16.0.

\section{DATA AVAILABILITY STATEMENT}

All datasets generated for this study are included in the article/supplementary material. 


\section{ETHICS STATEMENT}

The studies involving human participants were reviewed and approved by Institutional Review Board (NIRTIEC2010007) of National Institute for Research in Tuberculosis (NIRT), Chetpet, Chennai, Tamil Nadu, India. The patients/participants provided their written informed consent to participate in this study.

\section{AUTHOR CONTRIBUTIONS}

GK and SB conceived and designed the experiments, analyzed the data, and wrote the paper. GK, NK, and KM performed the experiments. DB, RS, DN, VB, and SB contributed materials, reagents, analysis tools.

\section{REFERENCES}

1. Dye C, Williams BG. The population dynamics and control of tuberculosis. Science. (2010) 328:856-61. doi: 10.1126/science.1185449

2. Sanou A, Bañuls A-L, van Anh NT, Godreuil S. Mycobacterium tuberculosis: ecology and evolution of a human bacterium. J Med Microbiol. (2015) 64:1261-9. doi: 10.1099/jmm.0.000171

3. Zumla A, Raviglione M, Hafner R, von Reyn CF. Tuberculosis. N Engl J Med. (2013) 368:745-55. doi: 10.1056/NEJMra1200894

4. Al Otaibi F, El Hazmi MM. Extra-pulmonary tuberculosis in Saudi Arabia. Indian J Pathol Microbiol. (2010) 53:227-31. doi: 10.4103/0377-4929.64327

5. Shafer RW, Edlin BR. Tuberculosis in patients infected with human immunodeficiency virus: perspective on the past decade. Clin Infect Dis. (1996) 22:683-704. doi: 10.1093/clinids/22.4.683

6. Zar HJ, Tannenbaum E, Apolles P, Roux P, Hanslo D, Hussey G. Sputum induction for the diagnosis of pulmonary tuberculosis in infants and young children in an urban setting in South Africa. Arch Dis Child. (2000) 82:3058. doi: 10.1136/adc.82.4.305

7. World Health Organization. Global Tuberculosis Report. (2019). Available online at: https://www.who.int/tb/publications/global_report/en/

8. World Health Organization. The Use of Lateral Flow Urine Lipoarabinomannan Assay (Lf-Lam) For The Diagnosis Screening of Active Tuberculosis In People Living With HIV. Geneva: WHO (2015).

9. Yerlikaya S, Broger T, MacLean E, Pai M, Denkinger CM. A tuberculosis biomarker database: the key to novel TB diagnostics. Int J Infect Dis. (2017) 56:253-7. doi: 10.1016/j.ijid.2017.01.025

10. Wallis RS, Kim P, Cole S, Hanna D, Andrade BB, Maeurer M, et al. Tuberculosis biomarkers discovery: developments, needs, and challenges. Lancet Infect Dis. (2013) 13:362-72. doi: 10.1016/S1473-3099(13)70034-3

11. World Health Organization. The End TB Strategy. Geneva: WHO (2015).

12. Kik SV, Denkinger CM, Casenghi M, Vadnais C, Pai M. Tuberculosis diagnostics: which target product profiles should be prioritised? Eur Respir J. (2014) 44:537-40. doi: 10.1183/09031936.000 27714

13. Saunders BM, Cooper AM. Restraining mycobacteria: role of granulomas in mycobacterial infections. Immunol Cell Biol. (2000) 78:334-41. doi: 10.1046/j.1440-1711.2000.00933.x

14. Klein T, Bischoff R. Active metalloproteases of the disintegrin and metalloprotease (ADAM) family: biological function and structure. JProteome Res. (2011) 10:17-33. doi: 10.1021/pr100556z

15. Klein T, Bischoff R. Physiology and pathophysiology of matrix metalloproteases. Amino Acids. (2011) 41:27190. doi: 10.1007/s00726-010-0689-x

16. Brew K, Nagase H. The tissue inhibitors of metalloproteinases (TIMPs): an ancient family with structural and functional diversity. Biochim Biophys Acta. (2010) 1803:55-71. doi: 10.1016/j.bbamcr.2010.01.003

\section{FUNDING}

This research work was supported by the Division of Intramural Research, National Institute of Allergy and Infectious Diseases (NIAID), National Institute of Health.

\section{ACKNOWLEDGMENTS}

The authors immensely thank V. Rajesh Kumar of NIHNIRT-ICER and the other staff members of Department of Clinical Research, NIRT, Government Stanley Hospital, Government General Hospital and Government Kilpauk Medical Hospital, Chennai, Tamil Nadu for valuable assistance in patient recruitment. We also thank Kannan Thiruvengadam of NIRT for his help in performing logistic regression analysis.

17. Ugarte-Gil CA, Elkington P, Gilman RH, Coronel J, Tezera LB, Bernabe-Ortiz A, et al. Induced sputum MMP-1, $-3 \&-8$ concentrations during treatment of tuberculosis. PLoS ONE. (2013) 8:e61333. doi: 10.1371/journal.pone.0061333

18. Sundararajan S, Babu S, Das SD. Comparison of localized versus systemic levels of Matrix metalloproteinases (MMPs), its tissue inhibitors (TIMPs) and cytokines in tuberculous and non-tuberculous pleuritis patients. Hum Immunol. (2012) 73:985-91. doi: 10.1016/j.humimm.2012.07.042

19. Lavrova AI, Esmedljaeva DS, Belik V, Postnikov EB. Matrix metalloproteinases as markers of acute inflammation process in the pulmonary tuberculosis. Data. (2019) 4:137. doi: 10.3390/data4040137

20. Rohlwink UK, Walker NF, Ordonez AA, Li YJ, Tucker EW, Elkington PT et al. Matrix metalloproteinases in pulmonary and central nervous system tuberculosis-A review. Int J Mol Sci. (2019) 20:1350. doi: 10.3390/ijms20061350

21. Stek C, Allwood B, Walker NF, Wilkinson RJ, Lynen L, Meintjes G. The immune mechanisms of lung parenchymal damage in tuberculosis and the role of host-directed therapy. Front Microbiol. (2018) 9:2603. doi: 10.3389/fmicb.2018.02603

22. Hoheisel G, Sack U, Hui DS, Huse K, Chan KS, Chan KK, et al. Occurrence of matrix metalloproteinases and tissue inhibitors of metalloproteinases in tuberculous pleuritis. Tuberculosis. (2001) 81:203-9. doi: 10.1054/tube.2000.0276

23. Sheen P, O'Kane CM, Chaudhary K, Tovar M, Santillan C, Sosa J, et al. High MMP-9 activity characterises pleural tuberculosis correlating with granuloma formation. Eur Respir J. (2009) 33:134-41. doi: 10.1183/09031936.00127807

24. Park KJ, Hwang SC, Sheen SS, Oh YJ, Han JH, Lee KB. Expression of matrix metalloproteinase-9 in pleural effusions of tuberculosis and lung cancer. Respiration. (2005) 72:166-75. doi: 10.1159/000084048

25. Singh S, Kubler A, Singh UK, Singh A, Gardiner H, Prasad R, et al. Antimycobacterial drugs modulate immunopathogenic matrix metalloproteinases in a cellular model of pulmonary tuberculosis. Antimicrob Agents Chemother. (2014) 58:4657-65. doi: 10.1128/AAC.02141-13

26. Thuong NT, Dunstan SJ, Chau TT, Thorsson V, Simmons CP, Quyen NT, et al. Identification of tuberculosis susceptibility genes with human macrophage gene expression profiles. PLoS Pathog. (2008) 4:e1000229. doi: 10.1371/journal.ppat.1000229

27. Kim MJ, Wainwright HC, Locketz M, Bekker LG, Walther GB, Dittrich C, et al. Caseation of human tuberculosis granulomas correlates with elevated host lipid metabolism. EMBO Mol Med. (2010) 2:258-74. doi: 10.1002/emmm.201000079

28. Mehra S, Pahar B, Dutta NK, Conerly CN, Philippi-Falkenstein $\mathrm{K}$, Alvarez $\mathrm{X}$, et al. Transcriptional reprogramming in nonhuman primate (rhesus macaque) tuberculosis granulomas. PLoS ONE. (2010) 5:e12266. doi: 10.1371/journal.pone.0012266

29. Kübler A, Luna B, Larsson C, Ammerman NC, Andrade BB, Orandle M, et al. Mycobacterium tuberculosis dysregulates MMP/TIMP balance to drive 
rapid cavitation and unrestrained bacterial proliferation. J Pathol. (2015) 235:431-44. doi: 10.1002/path.4432

30. Andrade BB, Pavan Kumar N, Amaral EP, Riteau N, Mayer-Barber KD, Tosh KW, et al. Heme oxygenase-1 regulation of matrix metalloproteinase1 expression underlies distinct disease profiles in tuberculosis. J Immunol. (2015) 195:2763-73. doi: 10.4049/jimmunol.1500942

31. Coussens AK, Wilkinson RJ, Nikolayevskyy V, Elkington PT, Hanifa Y, Islam $\mathrm{K}$, et al. Ethnic variation in inflammatory profile in tuberculosis. PLoS Pathog. (2013) 9:e1003468. doi: 10.1371/journal.ppat.1003468

32. Pavan Kumar N, Anuradha R, Andrade BB, Suresh N, Ganesh R, Shankar J, et al. Circulating biomarkers of pulmonary and extrapulmonary tuberculosis in children. Clin Vaccine Immunol. (2013) 20:704-11. doi: 10.1128/CVI.00038-13

33. Hibbs MS, Bainton DF. Human neutrophil gelatinase is a component of specific granules. J Clin Invest. (1989) 84:1395-402. doi: 10.1172/JCI1 14312

34. Walker NF, Wilkinson KA, Meintjes G, Tezera LB, Goliath R, Peyper JM, et al. Matrix degradation in human immunodeficiency virus type 1associated tuberculosis and tuberculosis immune reconstitution inflammatory syndrome: a prospective observational study. Clin Infect Dis. (2017) 65:12132. doi: $10.1093 / \mathrm{cid} / \mathrm{cix} 231$

35. Ravimohan S, Tamuhla N, Kung SJ, Nfanyana K, Steenhoff AP, Gross $\mathrm{R}$, et al. Matrix metalloproteinases in tuberculosis-immune reconstitution inflammatory syndrome and impaired lung function among advanced HIV/TB co-infected patients initiating antiretroviral therapy. EBio Med. (2015) 3:100-7. doi: 10.1016/j.ebiom.2015.11.040

36. Hrabec E, Strek M, Zieba M, Kwiatkowska S, Hrabec Z. Circulation level of matrix metalloproteinase- 9 is correlated with disease severity in tuberculosis patients. Int J Tuberc Lung Dis. (2002) 6:713-9.

37. Demedts IK, Morel-Montero A, Lebecque S, Pacheco Y, Cataldo D, Joos GF, et al. Elevated MMP-12 protein levels in induced sputum from patients with COPD. Thorax. (2006) 61:196-201. doi: 10.1136/thx.2005.042432

38. Quiding-Ja rbrink M, Smith DA, Bancroft GJ. Production of matrix metalloproteinases in response to mycobacterial infection. Infect Immun. (2001) 69:5661-70. doi: 10.1128/IAI.69.9.5661-5670.2001

39. Taylor JL, Hattle JM, Dreitz SA, Troudt JM, Izzo LS, Basaraba RJ, et al. Role for matrix metalloproteinase 9 in granuloma formation during pulmonary Mycobacterium tuberculosis infection. Infect Immun. (2006) 74:6135-44. doi: 10.1128/IAI.02048-05

40. Ong CW, Elkington PT, Brilha S, Ugarte-Gil C, Tome-Esteban MT, Tezera LB, et al. Neutrophil-derived MMP-8 drives AMPKdependent matrix destruction in human pulmonary tuberculosis. PLoS Pathog. (2015) 11:e1004917. doi: 10.1371/journal.ppat. 1004917

41. Parasa VR, Muvva JR, Rose JF, Braian C, Brighenti S, Lerm M. Inhibition of tissue matrix metalloproteinases interferes with Mycobacterium tuberculosisinduced granuloma formation and reduces bacterial load in a human lung tissue model. Front Microbiol. (2017) 8:2370. doi: 10.3389/fmicb.2017. 02370

42. Elkington PT, Shiomi T, Breen R, Nuttall RK, Ugarte-Gil CA, Walker NF, et al. MMP-1 drives immunopathology in human tuberculosis and transgenic mice. J Clin Invest. (2011) 121:1827-33. doi: 10.1172/JCI45666

43. Elkington PT, Emerson JE, Lopez-Pascua LD, O'Kane CM, Horncastle DE, Boyle JJ et al. Mycobacterium tuberculosis upregulates matrix metalloproteinase-1 secretion from human airway epithelial cells via a p38 MAPK switch. J Immunol. (2005) 175:5333-40. doi: 10.4049/jimmunol.175.8.5333

44. Leco KJ, Waterhouse P, Sanchez OH, Gowing KL, Poole AR, Wakeham A, et al. Spontaneous air space enlargement in the lungs of mice lacking tissue inhibitor of metalloproteinases-3 (TIMP-3). J Clin Invest. (2001) 108:81729. doi: 10.1172/JCI200112067

Conflict of Interest: The authors declare that the research was conducted in the absence of any commercial or financial relationships that could be construed as a potential conflict of interest.

Copyright (c) 2020 Kathamuthu, Kumar, Moideen, Nair, Banurekha, Sridhar, Baskaran and Babu. This is an open-access article distributed under the terms of the Creative Commons Attribution License (CC BY). The use, distribution or reproduction in other forums is permitted, provided the original author(s) and the copyright owner(s) are credited and that the original publication in this journal is cited, in accordance with accepted academic practice. No use, distribution or reproduction is permitted which does not comply with these terms. 